\title{
3D-QSAR predictions for bovine serum albumin-water partition coefficients of organic anions using quantum mechanically based descriptors
}

\section{Lukas Linden, Kai-Uwe Goss, Satoshi Endo}

\begin{tabular}{|c|l|}
\hline Citation & Environmental Science: Processes \& Impacts, 19(3); 261-269 \\
\hline Issue Date & 2017-03-01 \\
\hline Type & Journal Article \\
\hline Textversion & author \\
\hline $\begin{array}{c}\text { Supplementary } \\
\text { files }\end{array}$ & Supplementary information is available at https://doi.org/10.1039/C6EM00555A. \\
\hline Relation & $\begin{array}{l}\text { The following article has been accepted by Environmental Science: Processes } \\
\text { Impacts. After it is published, it will be found at } \\
\text { https://doi.org/10.1039/C6EM00555A }\end{array}$ \\
\hline DOI & \begin{tabular}{l}
$10.1039 / C 6 E M 00555 A$ \\
\hline
\end{tabular} \\
\hline
\end{tabular}

\author{
Self-Archiving by Author(s) \\ Placed on: Osaka City University
}

LINDEN, L., GOSS, K.-U., \& ENDO, S. (2017). 3D-QSAR predictions for bovine serum albuminwater partition coefficients of organic anions using quantum mechanically based descriptors. Electronic supplementary information (ESI) available. Environmental Science: Processes \& Impacts. 19, 261-269. https://doi.org/10.1039/C6EM00555A 
2 water partitioning coefficients of organic anions using

3 quantum mechanically based descriptors"

4

5 Lukas Linden ${ }^{a}$, Kai-Uwe Goss ${ }^{a, b}$, Satoshi Endo ${ }^{a, c *}$

7 a Helmholtz Centre for Environmental Research UFZ, Permoserstr. 15, D-04318

$8 \quad$ Leipzig, Germany

9 b University of Halle-Wittenberg, Institute of Chemistry, Kurt Mothes Str. 2, D1006120 Halle, Germany

11 c Osaka City University, Urban Research Plaza \& Graduate School of Engineering,

12 Sugimoto 3-3-138, Sumiyoshi-ku, 558-8585 Osaka, Japan

15 *corresponding author: phone/fax: ++81-6-6605-2763; e-mail:

16 satoshi.endo@urban.eng.osaka-cu.ac.jp 


\section{Abstract}

Ionic organic chemicals are a class of chemicals that is released in the environment in a large amount from anthropogenic sources. Among various chemical and biological processes, binding to serum albumin is particularly relevant for the toxicokinetic behavior of ionic chemicals. Several experimental studies showed that steric effects have a crucial influence on the sorption to bovine serum albumin (BSA). In this study, we investigated whether a 3D quantitative structure activity relationship (3D-QSAR) model can accurately account for these steric effects by predicting the BSA-water partition coefficients ( $K_{\mathrm{BSA} / \text { water }}$ ) of neutral and anionic organic chemicals. The 3D-QSAR tested here uses quantum mechanically derived local sigma profiles as descriptors. In general, the 3D-QSAR model was able to predict the partition coefficients of neutral and anionic chemicals with an acceptable quality (RMSE test set $0.63 \pm 0.10, \mathrm{R}^{2}$ test set $0.52 \pm 0.15$, both for $\log K_{\mathrm{BSA} / \text { water }}$. Particularly notable is that steric

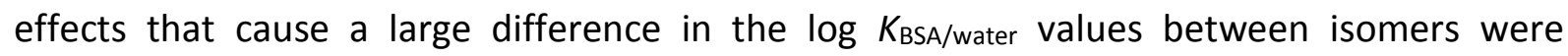
successfully reproduced by the model. The prediction of unknown $K_{\mathrm{BSA} / \text { water }}$ values with the proposed model should contribute to improved environmental and toxicological assessments of chemicals.

\section{Graphical Abstract}

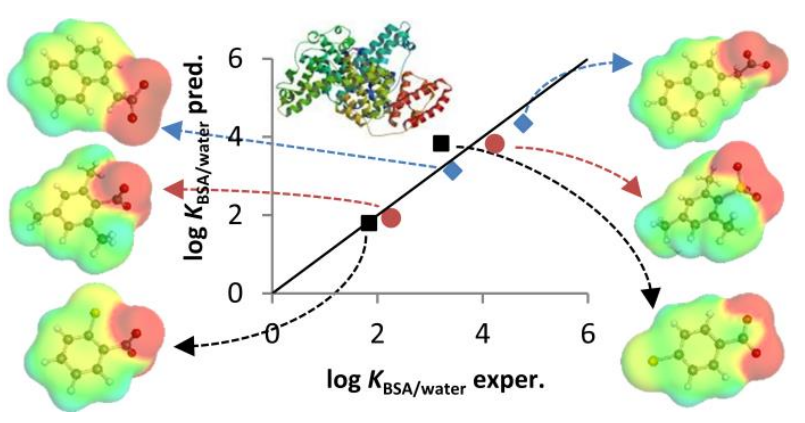




\section{Introduction}

Ionic organic chemicals are common types of chemicals in industry and our daily life. They are, among others, used as pesticides; e.g., 2,4-dichlorophenoxyacetic acid (2,4-D) and methylchlorophenoxypropionic acid (mecoprop) are among the most widely used herbicides $^{1,2}$ and both are anionic at typical environmental and physiological $\mathrm{pH}$. Many pharmaceuticals are also ionic; e.g., ibuprofen is an anionic chemical under neutral pH and is one of the most commonly taken nonsteroidal anti-inflammatory drugs ${ }^{3}$. The wide spread of ionic chemicals is also reflected in the general statistics, e.g., under REACH (the registration evaluation authorization and restriction of chemicals regulation of the European Union) around $50 \%$ of the preregistered chemicals are estimated to be ionogenic. ${ }^{4}$ Nevertheless, the ecotoxicological and environmental assessment of organic chemicals (including modeling of their fate) has its focus on neutral species and usually treats the ionic species in a simplistic manner, i.e., with the assumption that ionic species only occur in aqueous phases and do not partition into other phases. However, a number of experimental studies demonstrate that even a rather strong sorption of organic cations to natural organic matter and mineral surfaces in soils ${ }^{5}$ and of both cations and anions to phospholipids and proteins in biological tissues may occur ${ }^{6-10}$.

A biological phase particularly relevant for the toxicokinetic behavior of ionic chemicals is serum albumin, the most abundant blood protein of mammals and often a predominating sorption phase in blood. ${ }^{11}$ Through its relatively low specificity and strong binding for many chemicals, serum albumin influences the transport and the distribution of many organic ions in organisms. Particularly, anionic chemicals including perfluorinated alkyl acids ${ }^{12-13}$ and nonsteroidal anti-inflammatory drugs ${ }^{14}$ are known to bind strongly to serum albumin. It is also noted that fetal bovine serum is the most commonly used serum supplement for cell 
culture assays, where bovine serum albumin (BSA) has a strong impact on the freely dissolved concentration of the test chemical in the assays. ${ }^{15}$ Recently, Henneberger et al. published BSA/water partition coefficients $\left(K_{\mathrm{BSA} / \text { water }}\left[\mathrm{L}_{\text {water }} / \mathrm{kg}_{\mathrm{BSA}}\right]\right)$ for a broad set of ionic chemicals measured in a consistent condition. ${ }^{7} K_{\mathrm{BSA} / \text { water }}$ data help to assess the chemical distribution in organisms and bioassay systems. ${ }^{16}$ The reported ionic partition data to serum albumin show specific steric effects, which cannot easily be described by common methods for the prediction of partition coefficients such as polyparameter linear free energy relationships (pp-LFERs). ${ }^{17}$ Prediction of $K_{\mathrm{BSA} / \text { water }}$ may become even more challenging when one aims for a model that can be used both for neutral ${ }^{18}$ and ionic organic chemicals. In this study, we aim to construct a model that i) is capable of predicting log $K_{\mathrm{BSA} / \text { water }}$ of neutral and ionic chemicals, ii) can cover the specific 3D effects that influence the binding to BSA, and iii) can be used to estimate log $K_{\mathrm{BSA} / \text { water }}$ for the (eco)toxicological and environmental assessment of organic chemicals.

A modeling tool that is conceptually capable of predicting steric effects on sorption is the 3D quantitative structure activity relationship (3D-QSAR), which correlates 3D-structural features of the chemicals to the property of interest. This approach has been developed since the late $80 s^{19}$ and is a well-established ligand-based approach to generate a predictive model ${ }^{20}$. Recently, Klamt et al combined an existing 3D-QSAR method with quantum chemically-based molecular descriptors, the local sigma profiles (LSPs). ${ }^{21}$ The LSPs emerge from a solid theoretical basis, the COSMO-RS (conductor-like screening model for real solvents) method. ${ }^{22-23}$ The COSMO-RS method uses the COSMO surface polarization charge densities (also called sigma surface) to calculate, among others, partition coefficients and was successfully applied to numerous partition systems. ${ }^{24-25}$ The sigma surface describes the abilities of a molecule to undergo intermolecular interactions including electrostatic, 
hydrogen bond, and van der Waals interactions with its neighbor molecules. ${ }^{26}$ The COSMOsim3D method discretizes the sigma surface into LSPs. The LSPs are 4-dimensional histograms describing the amount of surface area with a certain sigma interval in a specific part of the molecule. ${ }^{21}$ Klamt et al. suggested that LSPs are theoretically more suitable for a linear regression model than the standard comparative molecular field analysis (CoMFA) descriptors $^{21}$, the latter use a van der Waals and an electrostatic potential derived from a molecular mechanics calculation ${ }^{27}$. LSPs were already applied by us to predict the binding to $\alpha$-cyclodextrin ${ }^{28}$, which is also influenced by $3 D$ effects ${ }^{29}$ and is a typical test system that shows specific binding ${ }^{30-31}$. LSPs resulted in a better prediction than the standard CoMFA descriptors for $\alpha$-cyclodextrin binding data ${ }^{28}$. In this study, we test whether steric effects that influence the partitioning to $\mathrm{BSA}^{7}$ can also be modeled by the LSPS.

\section{Methods}

\section{Data set}

Two data sets of $K_{\mathrm{BSA} / \text { water }}$ were combined in our study: the data set from Endo et al. ${ }^{18}$ with 83 neutral chemicals ( $\log K_{\mathrm{BSA} / \text { water }} 1.48-4.76$ ) and the data set from Henneberger et al. ${ }^{7}$ with

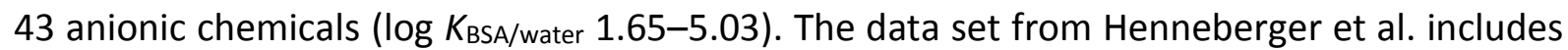
many benzoic acids anions and naphthoic acids anions with different substitutions and is thus suitable for investigating 3D structural effects on BSA binding. The four cationic chemicals from the Henneberger data set were not used in this work, because their number is too small for meaningful evaluation.

\section{D-QSAR}

The sorption to binding proteins such as BSA is influenced by the spatial structure of the sorption sites and any possible steric hindrance. This means that a modeling approach needs to represent the spatial structure and the chemical environment of the sorption sites. It 
should be noted that BSA has multiple binding sites and that the most favorable binding site may depend on the solutes. Thus, to apply 3D-QSARs for BSA binding constants, we have to set the working hypothesis that the different reported sorption sites of BSA are alike and that their spatial structure and interaction possibilities can be expressed through one characteristic binding site. ${ }^{32}$

In general, 3D-QSAR modeling takes the following steps: (1) 3D-structure generation for the sorbing chemicals, (2) alignment, (3) generation of independent variables, (4) training and test set selection in the experimental data set, (5) model generation by the training set with partial least square (PLS) regression analysis, and (6) model evaluation using the test set. Here, we used the method combination that performed the best in terms of the overall statistics and the qualitative descriptions in the previous publication for $\alpha$-cyclodextrin binding. ${ }^{28}$

\section{Local sigma profiles}

The LSPs are a spatial representation of the surface polarization charge densities and thereby of the interaction possibilities of a chemical (for a graphical explanation see Fig. SI 1). The LSPs are derived from the 3D-COSMO files of the chemicals. The LSPs can be used for the alignment of the chemicals and for the PLS regression model as independent variables. Each LSP was split in sections of $0.006 \mathrm{e} / \AA^{2}$ to capture the spatial distribution of surface segments with similar charge densities. LSP 1 starts with the most negative sigma value (in this work, 0.024 to $-0.018 \mathrm{e} / \AA^{2}$ ) (note that a negative sigma charge value corresponds to a positive partial charge and vice versa) and the LSP with the highest index (in this work, 10) represents the most positive sigma charge values of the molecular surface $\left(0.030-0.036 \mathrm{e} / \AA^{2}\right)$. 


\section{Alignment}

Prior to building a model, we had to generate a common binding hypothesis, i.e., a common 3D alignment, between the solutes and BSA. For this purpose, we chose those five chemicals from the experimental data sets with the strongest binding to BSA and the most rigid structure, i.e., chemicals with at least one conjugated two-ring aromatic structure, which reduces the degrees of freedom for the alignment. These five chemicals are referred to as template chemicals. 3D structures of one to ten conformers of all chemicals were generated with COSMOconfX15 in combination with Turbomole (v. 7.0$)^{33}$ that performs the quantum mechanics calculations generating 3D-COSMO files. For more details, see ${ }^{28}$. The software COSMOsim $3 \mathrm{D}^{34}$ generated an averaged sigma surface (including the $3 \mathrm{D}$ information) from the sigma surfaces of the five template chemicals, namely benzo[ $[g, h, i]$ perylene, chrysene, pyrene, naphthalene-2-sulfonate, and 2-naphthaleneacetate. This averaged sigma surface is assumed to describe the 3D interaction requirements of the BSA binding site and was used for the alignment of the chemicals of the data set. These five chemicals are a reasonable choice for the template because a high partition coefficient corresponds to a good interaction with BSA and rigid structure helps to delineate the binding site better than flexible structure. Obviously, choice of template chemicals is always limited through the data availability of binding chemicals, which may partially limit the domain of applicability of the resulting model. The 3D similarity between the averaged sigma surface of the five template chemicals and the sigma surface of each chemical was maximized through the translation and rotation of each chemical in the 3D space; conceptually, this corresponds to a search for the chemical's relative position that is optimal for interactions with BSA. This optimization procedure was carried out using a grid with a $0.5 \AA$ spacing. All conformers generated for each chemical were aligned. The conformer with the highest alignment score was selected 
153 for further modeling and if there were multiple conformers with the same alignment score,

154 then the conformer with the lowest internal energy was used.

155

156

157

158

159

160

161

162

163

164

165

166

167

168

169

170

171

172

173

174

175

\section{Independent variables}

The independent variables for the model are the LSPs, thus the amount of the surface area within a certain sigma charge interval and a space interval. The LSPs were derived at each grid point of a box with a grid spacing of $2 \AA$ and a size that includes a $5 \AA$ space around the chemicals. In the end, there were ten LSP intervals and 2730 grid points, which gave 27300 independent variables but on average 2910 active independent variables (variables whose values are unequal to zero). The number of independent variables was then reduced by an exclusion of variables that have a SD below a level of 0.1 among all training chemicals and a fractional factorial design selection ${ }^{35-36}$.

\section{Selection procedures for training and test sets}

The quality and the predictive power of the 3D-QSAR models were assessed with the test sets whose chemicals were not part of the respective training sets and thus did not influence the construction of the respective model. The statistical results of 3D-QSAR modeling depend highly on the combination of training and test sets. We decided to use several combinations of training and test sets (see the next paragraph) to capture this dependency and to obtain statistical results that represent the entire data set. The two phenolates in Henneberger's set, namely pentachlorophenolate and bromoxynil anion were used as additional validation chemicals, because we wanted to test how the model performs with the extrapolation to external data that are not represented in the training set in terms of the ionic functional group. In addition, 1-bromo-2-naphthoic acid also was also used for 
additional model validation in order to evaluate the model performance for an external test chemical that has the same ionic functional group as some of the test chemicals. It is worth noting that the critical settings of the alignment, i.e., the grid dimension and the choice of template chemicals, were defined before the selection of test and training set.

The test sets included eleven anionic and 21 neutral chemicals. These correspond to $25.6 \%$ and $25.3 \%$, respectively, of the data available. The data set was sorted according to the

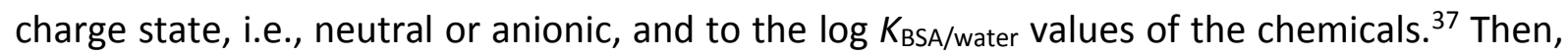
four consecutive chemicals with the same charge state were placed in one bin-the last bin of the ions contained five chemicals and the last bin of the neutral chemicals contained three chemicals (due to the fact that the total numbers of anionic and neutrals chemicals were not multiples of four). A random chemical from each bin was selected and placed in a test set and the rest of the chemicals were put in the training set; this was repeated until five test and training sets were generated. In addition to these random sets, modified test sets were generated by placing some structurally interesting chemicals (e.g., one chemical from a pair of isomers) always in the test set while the rest of the test sets was selected randomly. This was also performed five times. Modified test sets were prepared to study specific structural effects on $\log K_{\mathrm{BSA} / \text { water. }}$

\section{Statistical tool}

PLS regression analysis correlates the independent variables, i.e., the LSPs, to the dependent variables, i.e., the log $K_{\mathrm{BSA} / \text { water }}$ values, of the training set. For the PLS regression analysis the program Open3DQSAR ${ }^{38}$ was used, see reference ${ }^{28}$. Models with one to five PLS components (PCs) were generated and leave-two-out cross validation was used to select the best model in terms of predictive power and least chance of overfitting. This selected model is then used to predict the test set. 


\section{Domain of Applicability}

Tanimoto indices ${ }^{39}$ were applied to calculate the similarity of a test chemical against the training set. For the LSPs of two different chemicals ( $X$ and $Y$ ), the Tanimoto index is calculated as:

$$
T_{j}(x, y)=\frac{\sum X_{i j} Y_{i j}}{\sum X_{i j}^{2}+\sum Y_{i j}^{2}-\sum X_{i j} Y_{i j}}
$$

with $X_{i j}$ and $Y_{i j}$, the $j$-th field values at the $i$-th grid point. The arithmetic mean of the Tanimoto indices of the LSP 1 to 10 (i.e., the $j$-th field value in eq. 1 ) of a test chemical was calculated against each of the chemicals in the training set. Then, the mean of the five highest values was calculated (Tanimoto index mean). Data were grouped for every Tanimoto index mean value of 0.1 (called Tanimoto groups). We then compared the prediction errors of the different Tanimoto groups. The statistical difference between the variances of two Tanimoto groups was determined with a Brown-Forsythe analysis ${ }^{40}$ and the statistical difference between the medians of two Tanimoto groups was determined with a Mann-Whitney $U$ analysis ${ }^{41}$. These statistical tests were selected because the data are, most likely, not normally distributed.

\section{Results and Discussion}

\section{General performance of the models for $\log K_{\mathrm{BSA}} /$ water}

Five 3D-QSAR models were generated from different subsets of the available experimental data to describe the partitioning to BSA and to predict the respective test sets (using on average 230 independent variables). Fig. 1 gives examples of the test set predictions that resulted from different combinations of training and test sets. Panel A of Fig. 1 shows the best of the five predictions, while panel B shows the worst. All chemicals lay closer to the $1: 1$ line in panel A than in panel B. The biggest outlier of all predictions was flufenamic acid 
represent the mean \pm standard deviation). The neutral chemicals $(n=21)$ of the test set were

an RMSE of $0.68 \pm 0.23$. In general, the neutral chemicals are better predicted compared to neutral chemicals and 32 anionic chemical). However, the neutral chemicals in the calibration set appear to improve the description of the partitioning of anionic chemicals to BSA, as modelling using solely the anionic chemicals was less successful (data not shown)

chemicals.
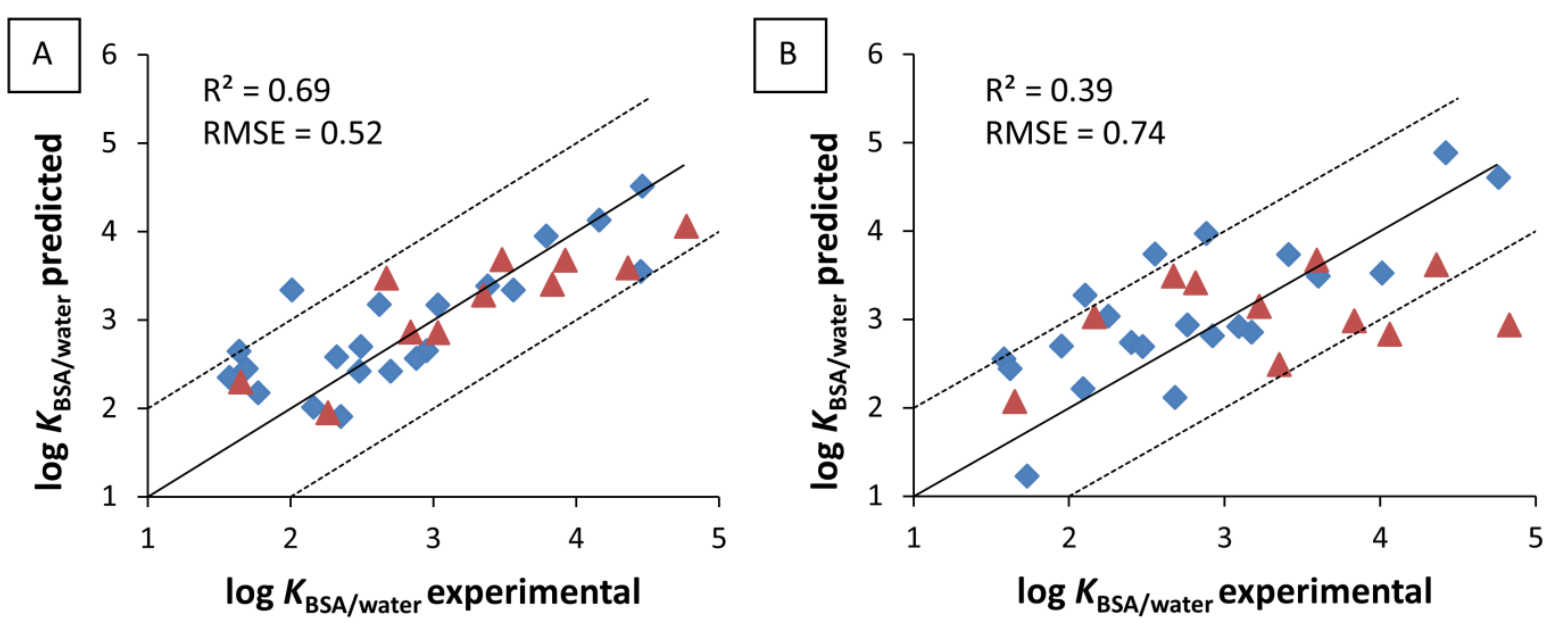

Figure 1 (A) Best and (B) worst prediction of log $K_{\mathrm{BSA} / \text { water }}$ of 21 neutral and 11 anionic chemicals of five random test sets. The blue diamonds indicate the neutral chemicals and the red triangles indicate the anionic chemicals. The solid line indicates the 1:1 line and the dashed lines indicate a deviation of 1 log unit from the 1:1 line.

A chance correlation of the models can be excluded based on the results of ten scrambling runs $^{42-43}$ using two log $K_{\mathrm{BSA} / \text { water }}$ sorted bins, i.e., the log $K_{\mathrm{BSA} / \text { water }}$ values of the chemicals of 
each bin were permuted in the respective bin prior to each run. The resulting statistics of

244 leave one out cross validation indicate non-predictive models (mean $R^{2}=0.44$, mean qLoo ${ }^{2}=$ $2450.002)$.

246 The binding mechanism behind the 3D-QSAR model can be examined with the help of the 247 contributions of the different LSPs to the overall model. Fig. 2 shows the percentage 248 contributions of the LSPs 2 to 9 to the PCs that were generated with the training set of the 249 best prediction (Fig. 1 A). The LSPs 1 and 10 contributed to the PCs only to a negligible 250 degree and thus are not shown. The LSP 8 (representing a part of the anionic interactions) 251 contributes to $20 \%$ to the PC 1 , which explains $48.7 \%$ of the variance in log $K_{\mathrm{BSA} / \text { water. Thus, }}$ 252 the positive influence of anionic partial charges on the partitioning to BSA, which is apparent 253 in the experimental data, is captured in the model. Other important interactions identified by the model are van der Waals interactions and the hydrophobic effect (LSPs 4, 5).

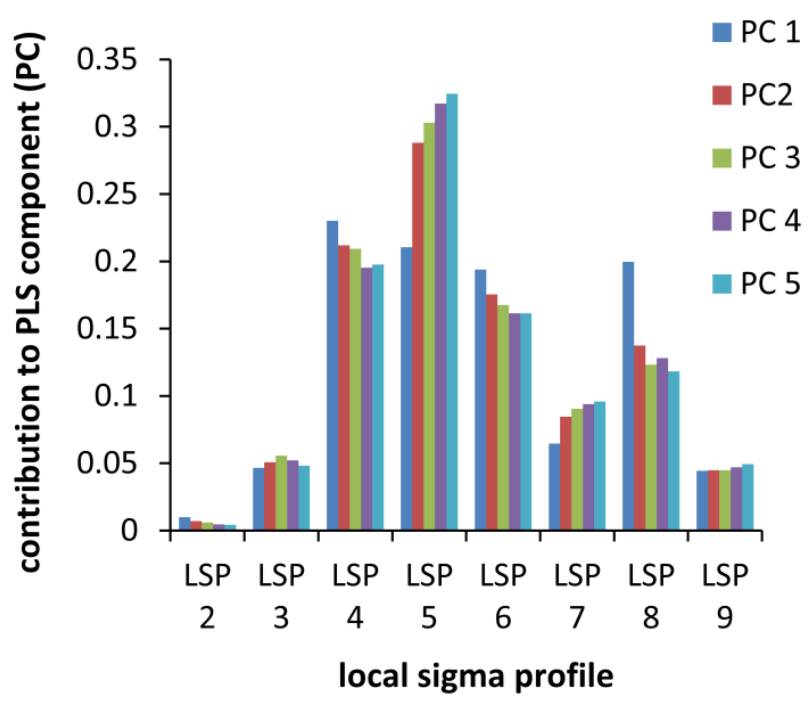
model. 


\section{Prediction of molecular steric effects}

259 For a further evaluation of the modeling approach, model performance was investigated for 260 isomer pairs using the modified test sets. In experimental data, several isomer pairs show 261 similar steric effects: an ortho-substitution of benzoate decreases log $K_{\mathrm{BSA}} /$ water substantially 262 compared to a para- or meta-substitution (2-chlorobenzoate vs. 4-chlorobenzoate, 2,6-dichlorobenzoate vs. 3,4-dichlorobenzoate, 2-methylbenzoate vs 4-methylbenzoate) and a substitution at the alpha-position of naphthalene decreases log $K_{\mathrm{BSA} / \text { water }}$ while a substitution at the beta-position increases $\log K_{\mathrm{BSA} / \text { water, }}$ particularly if the substitution group is negatively charged (1-naphthoic acid anion vs. 2-naphthoic acid anion, 1-naphthalenacetic acid anion vs. 2-naphthalenacetic acid anion). The steric hindrance by the ortho-substitution results in a twist of the carboxylate group ${ }^{7}$, which was speculated as a possible reason for the observed specificity. The relative sorption behavior of these isomer pairs with steric effects was predicted correctly by the models (Fig. 3). Even quantitative predictions (errors < 0.8) were achieved for three of the five isomer pairs. The other two had relatively large prediction errors: log $K_{\mathrm{BSA} / \text { water }}$ of 3,4-dichlorobenzoate is underestimated $(1.26 \pm 0.22 \log$

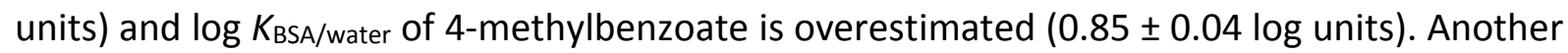
pair of chemicals that is of interest is 2,4,6-trimethylbenzene sulfonate and 2,4,6-

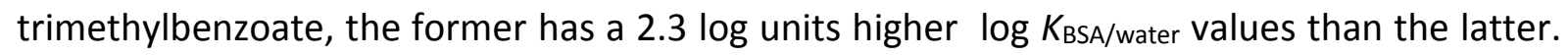

276 This difference is also predicted correctly but it might not be solely caused by the steric

277 hindrance of the carboxylate group, which is explained in the following. 

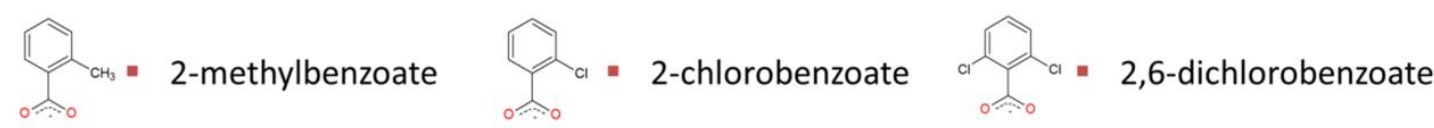

- 4-methylbenzoate
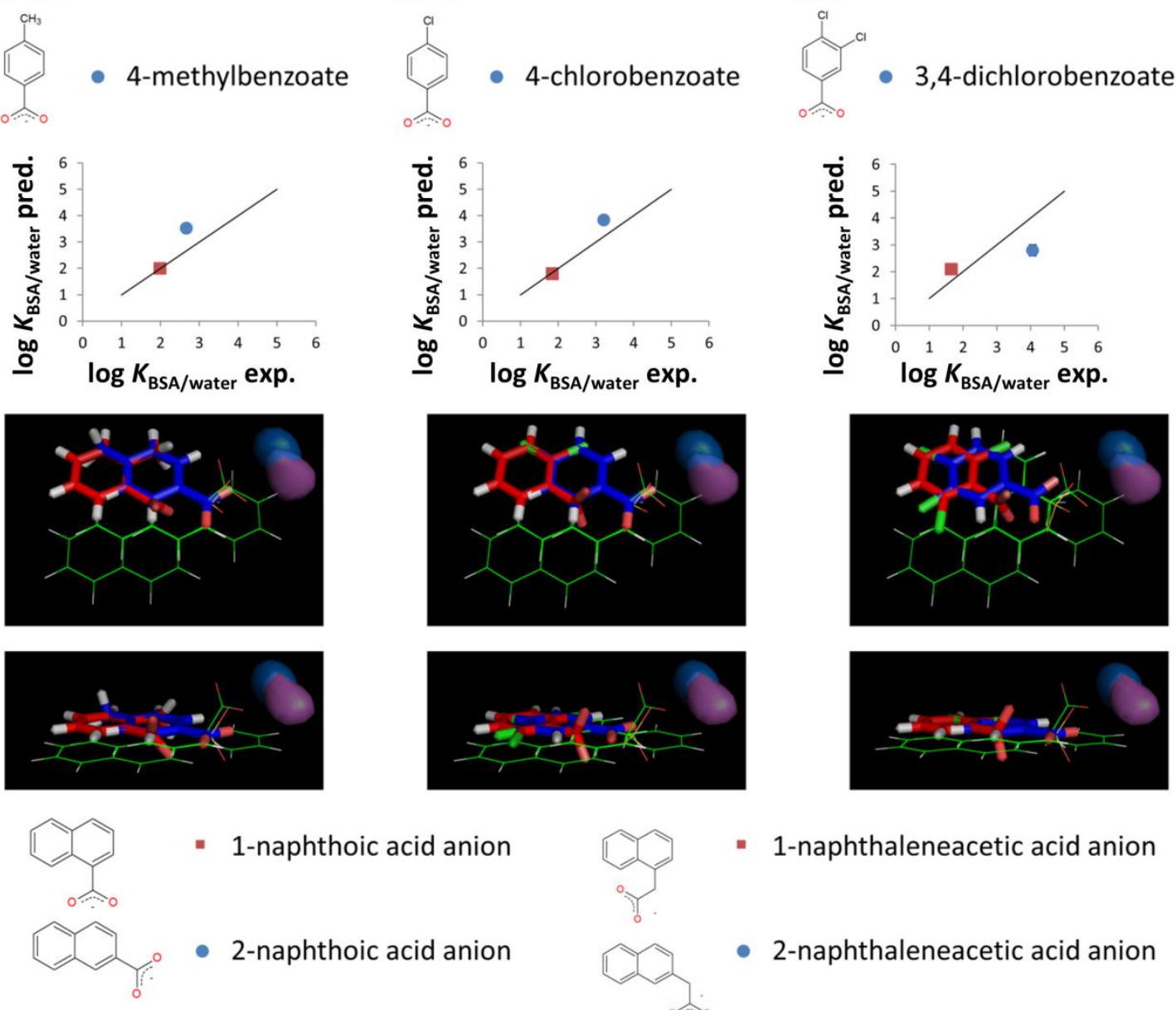

- 1-naphthoic acid anion

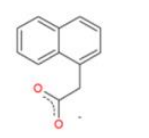

- 1-naphthaleneacetic acid anion

- 2-naphthoic acid anion

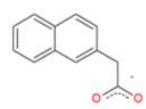

- 2-naphthaleneacetic acid anion
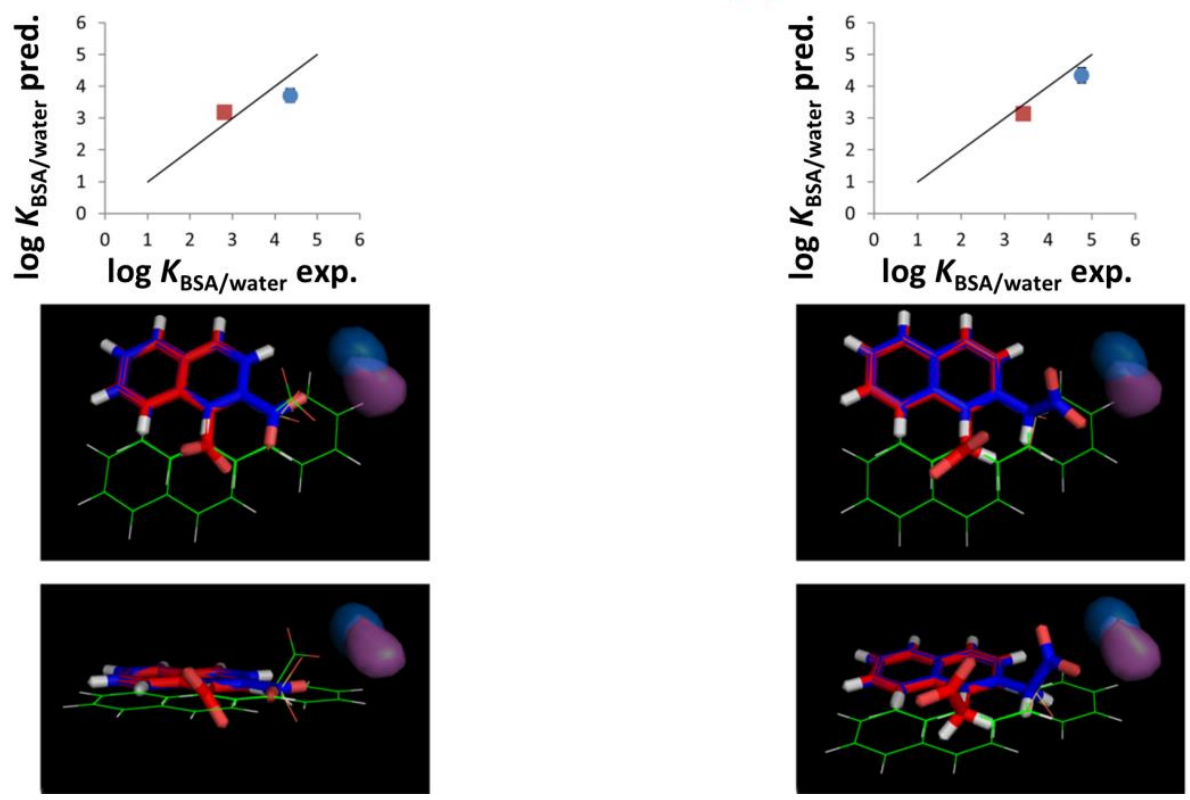

279 Figure 3 Experimental and the average predicted log $K_{\mathrm{BSA} / \text { water }}$ values of the modified test sets for several isomer pairs. The black line in the graphs indicates the 1:1 line, the red squares indicate the ortho- or alpha-substituted isomer, and the blue squares indicate the para- or beta-substituted isomer. The error bars indicate the respective standard deviation of the averaged predicted log 
$K_{\mathrm{BSA} / \text { water }}$ values (mostly not visible). The green lines in the pictures show the alignment chemicals/templates while the blue sticks show the ortho- or alpha-substituted isomer and the red sticks show the para- or beta-substituted isomer. The teal (LSP 7) and the violet (LSP 8) area indicate the space where the models identified a positive interaction of an anionic partial charge with BSA. The alignment figures were generated using Pymol. ${ }^{44}$

The alignment of the chemicals had an important role in the distinction of the isomer pairs

(Fig. 3). The green lines in the pictures of Fig. 3 show the five chemicals used as alignment template (see Methods, Alignment) while the sticks show the respective isomers. In addition, the anionic groups of naphthalene-2-sulfonate and 2-naphthaleneacetate are located at the same position, which could represent a possible interaction with a positively charged or electron-withdrawing group of BSA. ${ }^{45}$ Indeed, all isomers of Fig. 3 with the higher log $K_{\mathrm{BSA} / \text { water }}$ value have their charged group located close to this position (this interaction space is indicated in Fig. 3 by the teal and violet areas as it is expressed in the model). The isomers of Fig. 3 with the lower log $K_{\mathrm{BSA} / \text { water }}$ value (marked with red squares) have their anionic group at different positions, which seems to be inevitable for maximizing the overlapping of the rest of the structure to the template but seems to lead to omission of the interaction between the charged group of the chemical and BSA in the model. This difference in the positions of the anionic groups, which is caused by the twist of the carboxylate group, can

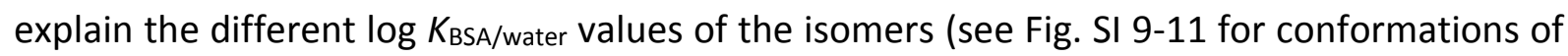
the isomers).

In comparison to the superimposition of the other aromatic chemicals, 2,4,6-trimethylbenzene sulfonate has a shifted position in the alignment (Fig. SI 3). This could be a hint for a different binding mode of 2,4,6-trimethylbenzene sulfonate (log $K_{\mathrm{BSA} / \text { water }}$ exper.: 4.23 pred.: 3.52 ). Possible causes of the difference include: a) the lower electron density of the aromatic ring of 2,4,6-trimethylbenzene sulfonate compared to the electron density of, for example, 2,4,6-trimethylbenzoate (log $K_{\mathrm{BSA} / \text { water }}$ exper.: 1.99 pred.: 
2.00), which could make an interaction with the $\pi$-system weaker ${ }^{45}$ in case of

2,4,6-trimethylbenzene sulfonate (Fig. SI 12 and 13) and thus enabling an interaction

between the charged sulfonate group and the BSA, because of b) the longer $\mathrm{C}_{-} \mathrm{SO}_{3}{ }^{-}$bond $(1.8 \AA)$ compared to the $\mathrm{C}-\mathrm{CO}_{2}^{-}$bond $(1.5 \AA)^{44}$, which might enable an interaction even in the presence of the steric hindrance of the neighboring methyl groups. Furthermore, the sulfonate group has higher interaction possibilities than the carboxylate group because the sulfonate group has an additional oxygen atom and the $\mathrm{C}-\mathrm{SO}_{3}{ }^{-}$bond is better rotatable than the $\mathrm{C}-\mathrm{CO}_{2}{ }^{-}$bond. Thus, the positions and interactions of the $\mathrm{sp}^{2}$ orbitals of the oxygens are more flexible in case of 2,4,6-trimethylbenzene sulfonate. These flexibilities of 2,4,6-trimethylbenzene sulfonate in the positioning and the interaction possibilities may result in a higher experimental and predicted log $K_{\mathrm{BSA} / \text { water }}$ value as compared to 2,4,6-trimethylbenzoate. These inferences are based on the alignment results, which led to successful modeling, but additional insight from further experimental data or direct modeling tools, like molecular dynamics simulation ${ }^{46}$, would be desirable.

\section{Domain of applicability}

The domain of applicability was assessed with the help of the Tanimoto indices. The median of the prediction errors for the five random test sets apparently decreases with increasing Tanimoto index mean (Fig. 4). This may suggest that the reliability of the prediction rises with increasing Tanimoto index mean. For statistical evaluation, we chose the second highest range of Tanimoto index mean $(0.60-0.70)$ as the reference group and tested the differences in prediction errors of all the other groups from it (Table SI 1). We did not consider the group $0.70-0.80$ because it comprises only four chemicals. Compared to the reference group, the median of the prediction errors is only significantly larger for the Tanimoto group of $0.30-0.40$. No group has a significantly different variance than the 
reference group. Note, however, that the prediction error depends strongly on the combination of test and training sets. We also compared predictions errors and Tanimoto index means using test and training sets generated by a slightly less random procedure. This procedure (see SI for details) uses each chemical once as a test set chemical. Although the resulting plot appears comparable to that presented in Fig. 4, the medians of the prediction errors are significantly larger for all Tanimoto groups $<0.50$ than for the reference group (Table SI 2), showing that the Tanimoto index means could indicate the domain of applicability. We do not know why Tanimoto index works in one case but not the other. Possible reasons include: The data size is not sufficiently large to show a statistical significance, and the Tanimoto index mean calculated this study (i.e., the mean of the top 5 Tanimoto indices) is not suitable.

The three anions that were not part of the model calibration set, nor included in Fig. 4, were used as additional validation chemicals. The prediction is accurate for 1-bromo-2-naphthoic acid anion (prediction error 0.08 log units) despite a relatively small Tanimoto index mean of 0.34. In contrast, bromoxynil anion and pentachlorophenolate were predicted with 2.47 and $2.33 \log$ units off, respectively. Both chemicals have a Tanimoto index mean value of 0.16 , which indicates a higher chance for a large prediction error. The large prediction errors for these two phenolates can be expected because the training set does not contain any phenolate, and their low Tanimoto index means reasonably explain the outlying behavior of these chemicals. In the alignment, bromoxynil anion and pentachlorophenolate are displaced compared to the other aromatic chemicals, which might be caused by the different nature of the anionic groups of the template chemicals and of these two phenolates. For a future successful prediction of $\log K_{\mathrm{BSA} / \text { water }}$ for phenolates two steps seems to be promising: i) an extended/different set of template chemicals which includes at least one phenolate and 
ii) more experimental data for phenolates and thus a better calibration through phenolates in the training set of the 3D-QSAR model.

Other chemicals that are expected to be out of the domain of applicability of the presented model are zwitterions and cations because they have no representation in the training set.

Multiply charged anions may also be difficult to predict because the effect of the second

362 charged group is probably not covered by the model. Other examples of chemicals that

363 should be out of the domain of applicability are big bulky chemicals (e.g., monensin

Tanimoto index mean 0.07, perfluorononanoic carboxylate Tanimoto index mean 0.09)

365 including oligosaccharides (e.g., maltotriose Tanimoto index mean 0.12), long tertiary and

quaternary organic chemicals (e.g., 4-butyl-4-pentylnonanal Tanimoto index mean 0.14),

367 because they are not part of the current calibration set and might bind to BSA through

368 another mechanism. The same holds true for fatty acids, which bind to a specific binding site

369 of $\mathrm{BSA}^{47}$ (e.g., undecane carboxylate Tanimoto index mean 0.19).

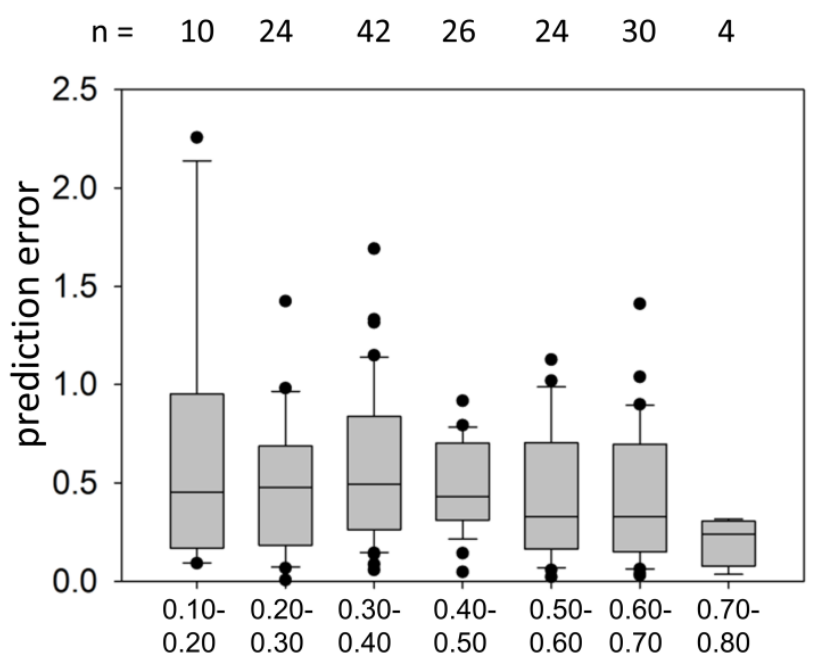

Figure 4 Prediction errors of the 3D-QSAR model plotted against the Tanimoto index range of the

372 five most similar chemicals of the training set. The boxes outline the $25^{\text {th }}$ to $75^{\text {th }}$ percentiles, the 373 lines through the centers represent the median, the whiskers indicate the $90^{\text {th }}$ and $10^{\text {th }}$ percentiles, and the dots indicate outlying points. The results for all five random test sets are plotted. 


\section{Conclusions}

The 3D-QSAR model with LSPs as descriptors is capable of describing and predicting log $K_{\mathrm{BSA} / \text { water }}$ for anionic and neutral chemicals. The assumptions behind the generated characteristic binding site (i.e., several localized binding sites with similar chemical environments and the interaction possibilities of the sites can be expressed as an averaged characteristic binding site) appear to be adequate for the 3D-QSAR modelling approach. The discrimination between different binding sites was not necessary for successful modeling for the data set used in this work. The steric effects that are responsible for up to two log units differences in $\log K_{\mathrm{BSA} / \text { water }}$ between structural isomers are successfully captured by the model. Thus, the model may be used for the prediction of unknown $K_{\mathrm{BSA} / \text { water }}$ for neutral and anionic chemicals, which is helpful for a qualified environmental and toxicological assessment of these chemicals. As an example, in an upcoming study the 3D-QSAR model developed in this work will be used to assess the freely dissolved concentration of chemicals in a typical cell assay. ${ }^{16}$ Furthermore, the model could contribute to an estimation of the bioaccumulation potential of organic anions, provided that other sorption phases such as phospholipid membranes are considered as well. Whereas serum albumin appears not to be the most important plasma binding protein for many cationic chemicals, ${ }^{48}$ an extension of the model with more cationic chemicals is still desirable because there are cations that bind strongly to serum albumin. ${ }^{11}$ An inclusion of zwitterions is another interesting example of possible extensions of the model applicability domain. The availability of accurately and consistently measured data will be the key to such future work.

\section{Acknowledgements}

The authors thank the Helmholtz Interdisciplinary Graduate School for Environmental Research (HIGRADE) for financial support. SE acknowledges the financial support from the 
constructive comments on the manuscript.

\section{Appendix A. Supplementary material}

Supplementary data associated with this article can be found, in the online version, at ...

\section{References}

1. Grube A, D. D., Kiely T, Wu L, Pesticides Industry Sales and Usage: 2006 and 2007 Market Estimates, United States Environmental Protection Agency, Washington, DC, 2011.

2. US Environmental Protection Agency, Reregistration Eligibility Decision for Mecoprop-p. https://archive.epa.gov/pesticides/reregistration/web/pdf/mcpp red.pdf, 2007.

3. IMS Health Rezeptfreie Schmerzmittel.
http://www.imshealth.de/files/web/Germany/Publikationen/Infografiken/2014 9 IMS Infografik \% 20Schmerzmittel.pdf (accessed 03.08.2016).

4. Franco, A.; Ferranti, A.; Davidsen, C.; Trapp, S., An unexpected challenge: ionizable compounds in the REACH chemical space, The International Journal of Life Cycle Assessment, 2010, 15 (4), 321-325.

5. Droge, S. T. J.; Goss, K.-U., Development and Evaluation of a New Sorption Model for Organic Cations in Soil: Contributions from Organic Matter and Clay Minerals, Environmental Science \& Technology, 2013, 47 (24), 14233-14241.

6. Bittermann, K.; Spycher, S.; Goss, K. U., Comparison of different models predicting the phospholipid-membrane water partition coefficients of charged compounds, Chemosphere, 2016, 144, 382-391.

7. Henneberger, L.; Goss, K.-U.; Endo, S., Equilibrium Sorption of Structurally Diverse Organic Ions to Bovine Serum Albumin, Environmental Science \& Technology, 2016, 50 (10), 5119-5126.

8. Ng, C. A.; Hungerbühler, K., Bioconcentration of Perfluorinated Alkyl Acids: How Important Is Specific Binding?, Environmental Science \& Technology, 2013, 47 (13), 7214-7223.

9. Henneberger, L.; Goss, K.-U.; Endo, S., Partitioning of Organic Ions to Muscle Protein: Experimental Data, Modeling, and Implications for in Vivo Distribution of Organic Ions, Environmental Science \& Technology, 2016, 50 (13), 7029-7036.

10. Kremer, J. M.; Wilting, J.; Janssen, L. H., Drug binding to human alpha-1-acid glycoprotein in health and disease, Pharmacological Reviews, 1988, 40 (1), 1-47.

11. Kragh-Hansen, U., Molecular aspects of ligand binding to serum albumin, Pharmacological Reviews, 1981, 33 (1), 17-53.

12. Bischel, H. N.; MacManus-Spencer, L. A.; Luthy, R. G., Noncovalent Interactions of Long-Chain Perfluoroalkyl Acids with Serum Albumin, Environmental Science \& Technology, 2010, 44 (13), 52635269.

13. Bischel, H. N.; MacManus-Spencer, L. A.; Zhang, C.; Luthy, R. G., Strong associations of shortchain perfluoroalkyl acids with serum albumin and investigation of binding mechanisms, Environmental Toxicology and Chemistry, 2011, 30 (11), 2423-2430.

14. Lapicque, F.; Muller, N.; Payan, E.; Dubois, N.; Netter, P., Protein Binding and Stereoselectivity of Nonsteroidal Anti-Inflammatory Drugs, Clinical Pharmacokinetics, 1993, 25 (2), 115-125.

15. Gülden, M.; Mörchel, S.; Tahan, S.; Seibert, H., Impact of protein binding on the availability and cytotoxic potency of organochlorine pesticides and chlorophenols in vitro, Toxicology, 2002, 175 (1-3), 201-213. 
16. Fischer, F.; Henneberger, L.; König, M.; Bittermann, K.; Linden, L.; Goss, K. U.; Escher, B. I., Modelling freely dissolved and internal cellular effect concentrations in the Tox21 in vitro bioassays, in Preparation, 2016.

17. Endo, S.; Goss, K. U., Applications of Polyparameter Linear Free Energy Relationships in Environmental Chemistry, Environmental Science \& Technology, 2014, 48 (21), 12477-12491.

18. Endo, S.; Goss, K.-U., Serum Albumin Binding of Structurally Diverse Neutral Organic Compounds: Data and Models, Chemical Research in Toxicology, 2011, 24 (12), 2293-2301.

19. Cramer, R. D.; Patterson, D. E.; Bunce, J. D., Comparative molecular field analysis (CoMFA). 1. Effect of shape on binding of steroids to carrier proteins, Journal of the American Chemical Society, 1988, 110 (18), 5959-5967.

20. Lambrinidis, G.; Vallianatou, T.; Tsantili-Kakoulidou, A., In vitro, in silico and integrated strategies for the estimation of plasma protein binding. A review, Advanced Drug Delivery Reviews, 2015, 86, 27-45.

21. Klamt, A.; Thormann, M.; Wichmann, K.; Tosco, P., COSMOsar3D: Molecular Field Analysis Based on Local COSMO б-Profiles, Journal of Chemical Information and Modeling, 2012, 52 (8), 2157 2164.

22. Klamt, A., Conductor-like Screening Model for Real Solvents: A New Approach to the Quantitative Calculation of Solvation Phenomena, The Journal of Physical Chemistry, 1995, 99 (7), 2224-2235.

23. Klamt, A.; Jonas, V.; Bürger, T.; Lohrenz, J. C. W., Refinement and Parametrization of COSMORS, The Journal of Physical Chemistry A, 1998, 102 (26), 5074-5085.

24. Klamt, A.; Eckert, F.; Arlt, W., COSMO-RS: An Alternative to Simulation for Calculating Thermodynamic Properties of Liquid Mixtures, Annual Review of Chemical and Biomolecular Engineering, 2010, 1 (1), 101-122.

25. Diedenhofen, M.; Klamt, A., COSMO-RS as a tool for property prediction of IL mixtures-A review, Fluid Phase Equilibria, 2010, 294 (1-2), 31-38.

26. Klamt, A., The COSMO and COSMO-RS solvation models, Wiley Interdisciplinary Reviews: Computational Molecular Science, 2011, 1 (5), 699-709.

27. Melo, C. C.; Braga, R. C.; Andrade, C. H., 3D-QSAR Approaches in Drug Design: Perspectives to Generate Reliable CoMFA Models, Curr. Comput.-Aided Drug Des., 2014, 10 (2), 148-159.

28. Linden, L.; Goss, K. U.; Endo, S., 3D-QSAR Predictions for $\alpha$-Cyclodextrin Binding Constants Using Quantum Mechanically Based Descriptors, Manuscript submitted for publication, 2016.

29. Linden, L.; Goss, K.-U.; Endo, S., Exploring 3D structural influences of aliphatic and aromatic chemicals on $\alpha$-cyclodextrin binding, Journal of Colloid and Interface Science, 2016, 468, 42-50.

30. Tabushi, I., Cyclodextrin catalysis as a model for enzyme action, Accounts of Chemical Research, 1982, 15 (3), 66-72.

31. Schneider, H. J., Binding mechanisms in supramolecular complexes, Angewandte Chemie, 2009, 48 (22), 3924-77.

32. Abou-Zied, O. K.; Al-Lawatia, N.; Elstner, M.; Steinbrecher, T. B., Binding of Hydroxyquinoline Probes to Human Serum Albumin: Combining Molecular Modeling and Förster's Resonance Energy Transfer Spectroscopy to Understand Flexible Ligand Binding, The Journal of Physical Chemistry B, 2013, 117 (4), 1062-1074.

33. Sijm, D.; Kraaij, R.; Belfroid, A., Environ. Pollut., 2000, 108 (1), 113.

34. Thormann, M.; Klamt, A.; Wichmann, K., COSMOsim3D: 3D-Similarity and Alignment Based on COSMO Polarization Charge Densities, Journal of Chemical Information and Modeling, 2012,52 (8), 2149-2156.

35. Baroni, M.; Clementi, S.; Cruciani, G.; Costantino, G.; Riganelli, D.; Oberrauch, E., Predictive ability of regression models. Part II: Selection of the best predictive PLS model, J. Chemom., 1992,6 (6), 347-356.

36. Baroni, M.; Costantino, G.; Cruciani, G.; Riganelli, D.; Valigi, R.; Clementi, S., Generating Optimal Linear PLS Estimations (GOLPE): An Advanced Chemometric Tool for Handling 3D-QSAR Problems, Quant. Struct.-Act. Relat., 1993, 12 (1), 9-20. 
37. Kauffman, G. W.; Jurs, P. C., QSAR and k-Nearest Neighbor Classification Analysis of Selective Cyclooxygenase-2 Inhibitors Using Topologically-Based Numerical Descriptors, Journal of Chemical Information and Computer Sciences, 2001, 41 (6), 1553-1560.

499 38. Tosco, P.; Balle, T., Open3DQSAR: a new open-source software aimed at high-throughput chemometric analysis of molecular interaction fields, J Mol Model, 2011, 17 (1), 201-208.

501

502

503

504

505

506

507

508

509

510

511

512

513 39. Monev, V., Introduction to similarity searching in chemistry, MATCH Commun. Math. Comput. Chem, 2004, 51, 7-38.

40. Brown, M. B.; Forsythe, A. B., Robust Tests for the Equality of Variances, Journal of the American Statistical Association, 1974, 69 (346), 364-367.

41. Mann, H. B.; Whitney, D. R., On a Test of Whether one of Two Random Variables is Stochastically Larger than the Other, 1947, 50-60.

42. Tropsha, A.; Gramatica, P.; Gombar, V. K., The Importance of Being Earnest: Validation is the Absolute Essential for Successful Application and Interpretation of QSPR Models, QSAR \& Combinatorial Science, 2003, 22 (1), 69-77.

43. Rücker, C.; Rücker, G.; Meringer, M., y-Randomization and Its Variants in QSPR/QSAR, Journal of Chemical Information and Modeling, 2007, 47 (6), 2345-2357.

44. Schrodinger, LLC, The PyMOL Molecular Graphics System, Version 1.3r1. 2010.

45. Peters Jr, T., All About Albumin. In All About Albumin, Academic Press: San Diego, 1995; pp $\mathrm{xV}$-xvii.

46. Sudhamalla, B.; Gokara, M.; Ahalawat, N.; Amooru, D. G.; Subramanyam, R., Molecular Dynamics Simulation and Binding Studies of $\beta$-Sitosterol with Human Serum Albumin and Its Biological Relevance, The Journal of Physical Chemistry B, 2010, 114 (27), 9054-9062.

518 47. van der Vusse, G. J., Albumin as Fatty Acid Transporter, Drug Metabolism and 519 Pharmacokinetics, 2009, 24 (4), 300-307.

520 48. Trainor, G. L., The importance of plasma protein binding in drug discovery, Expert Opinion on Drug Discovery, 2007, 2 (1), 51-64. 